Synthesis of few-layered graphene by ion implantation of carbon in nickel thin films

This article has been downloaded from IOPscience. Please scroll down to see the full text article.

2011 Nanotechnology 22085601

(http://iopscience.iop.org/0957-4484/22/8/085601)

View the table of contents for this issue, or go to the journal homepage for more

Download details:

IP Address: 129.104.38.6

The article was downloaded on 18/01/2011 at 08:13

Please note that terms and conditions apply. 


\title{
Synthesis of few-layered graphene by ion implantation of carbon in nickel thin films
}

\author{
Laurent Baraton ${ }^{1}$, Zhanbing He ${ }^{1}$, Chang Seok Lee ${ }^{1}$, \\ Jean-Luc Maurice ${ }^{1}$, Costel Sorin Cojocaru ${ }^{1}$, \\ Anne-Françoise Gourgues-Lorenzon ${ }^{2}$, Young Hee Lee $^{3}$ and \\ Didier Pribat ${ }^{3}$ \\ ${ }^{1}$ Laboratoire de Physique des Interfaces et des Couches Minces, CNRS UMR 7647, Ecole \\ Polytechnique, 91128, Palaiseau, France \\ ${ }^{2}$ Centre des Matériaux, Mines ParisTech, CNRS UMR 7633, BP 87, 91000, Évry Cedex, \\ France \\ ${ }^{3}$ BK 21, Physics Division, Department of Energy Science, Sungkyunkwan University, \\ Suwon 440-746, Korea \\ E-mail: didier53@skku.edu
}

Received 17 September 2010, in final form 19 September 2010

Published 17 January 2011

Online at stacks.iop.org/Nano/22/085601

\begin{abstract}
The synthesis of few-layered graphene is performed by ion implantation of carbon species in thin nickel films, followed by high temperature annealing and quenching. Although ion implantation enables a precise control of the carbon content and of the uniformity of the in-plane carbon concentration in the Ni films before annealing, we observe thickness non-uniformities in the synthesized graphene layers after high temperature annealing. These non-uniformities are probably induced by the heterogeneous distribution/topography of the graphene nucleation sites on the Ni surface. Taken altogether, our results indicate that the number of graphene layers on top of $\mathrm{Ni}$ films is controlled by the nucleation process on the $\mathrm{Ni}$ surface rather than by the carbon content in the Ni film.
\end{abstract}

(Some figures in this article are in colour only in the electronic version)

Graphene is the first stable two-dimensional crystal, discovered in 2004 [1]. Since then, this new nanomaterial has been the object of a large interest due to its peculiar and intriguing properties $[2,3]$. Low crystalline quality graphene is already used for several applications, such as composite materials and electrodes for batteries [4]. However, as far as electronic applications are concerned, high quality graphene films are needed as well as robust and reproducible methods to synthesize them.

The first method used to obtain graphene flakes was the mechanical exfoliation of highly oriented pyrolytic graphite (HOPG) [1], which produces high quality crystals with their dimensions in the $10-100 \mu \mathrm{m}$ range. Although not amenable to large areas and high throughputs, this method has been extensively used to demonstrate the amazing electronic properties of graphene [1-3]. The other early route for the synthesis of graphene films is the epitaxial graphitization of silicon carbide substrates [5]. This technique which has been refined recently [6] now yields graphene domains of $\sim 50 \mu \mathrm{m} \times 3 \mu \mathrm{m}$ on the terraces of monocrystalline
SiC substrates [6]. More recently, the epitaxial growth of graphene films on monocrystalline transition metals such as $\mathrm{Ru}(0001)$ has been revisited [7], producing larger graphene domains, with their dimensions in the $200 \mu \mathrm{m}$ range. A number of recent papers have also shown the interest of 'polycrystalline' graphene growth on non-carbide forming polycrystalline transition metals by decomposition of a carbon bearing gas precursor, in a way similar to chemical vapor deposition (CVD) [8-13]. Here, the idea is to produce graphene domains $(\sim 10 \mu \mathrm{m} \times 10 \mu \mathrm{m}$ or more [14]), larger than the grain size of the supporting metal films or foils and with low defect boundaries. However, this goal seems to be out of reach presently, since the domain size of single layer graphene grown by CVD on monocrystalline (111)-oriented $\mathrm{Cu}$ substrates is only of the order of a few $100 \mathrm{~nm}$ in both dimensions at best [15] and it is hard to imagine that growth on polycrystalline $\mathrm{Cu}$ would yield larger domains.

For those metals in which carbon has relatively high solubilities (e.g., $\mathrm{Ni}, \mathrm{Co}, \mathrm{Ru}, \ldots$ ), the metal-assisted CVD mechanism usually involves (i) catalytic decomposition of the 


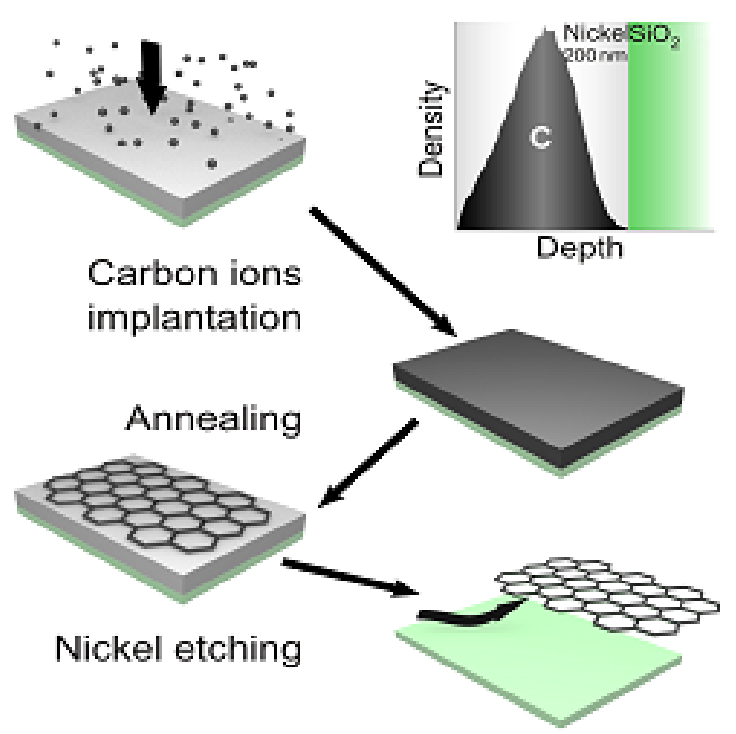

Figure 1. Schematic representation of the procedure used in this work. The inset shows the calculated carbon distribution through the thickness of the Ni film after ion implantation at an energy of $80 \mathrm{keV}$ (see [16] for details).

carbon bearing gas molecules on the metal surface, followed by (ii) dissolution of the released carbon atoms into the metal at high temperature $\left(900-1000^{\circ} \mathrm{C}\right)$ and (iii) precipitation of graphene onto the metal surface, either at the growth temperature when the solid solution has been saturated, or during cooling, because of the decrease of carbon solubility in the metal. However, for graphene films grown on $\mathrm{Ni}$, it seems difficult to precisely control the number of graphene layers over large areas [14]. When the solubility of carbon in the supporting metal is negligible (e.g., $\mathrm{Cu}$ ), graphene growth proceeds through a surface mechanism which is selflimiting, yielding films with essentially one monolayer of graphene [13, 14].

In this paper, we explore the possibility to grow graphene on polycrystalline $\mathrm{Ni}$ thin films by using ion implantation of carbon ions. The carbon density in one graphene layer is $\sim 3.8 \times 10^{15}$ atoms $\mathrm{cm}^{-2}$, corresponding to a dose easily accessible to ion implantation. Moreover, ion implantation is a robust technique, developed over the past $\sim 40$ years by the microelectronics industry, which could become a method of choice for graphene synthesis. Figure 1 schematically shows the procedure followed in this work. We have used $200 \mathrm{~nm}$ thick nickel films deposited by e-beam evaporation on oxidized silicon wafers $\left(\mathrm{SiO}_{2}\right.$ thickness $\left.\sim 300 \mathrm{~nm}\right)$. Carbon implantation in those $\mathrm{Ni}$ films was performed at an energy of $80 \mathrm{keV}$ (yielding a $\sim 100 \mathrm{~nm}$ projected range [16]), using a dose of $16 \times 10^{15} \mathrm{~cm}^{-2}$ which corresponds to the equivalent of $\sim 4$ graphene layers assuming a uniform precipitation of the total implanted $\mathrm{C}$ upon cooling and also assuming that $\mathrm{C}$ precipitation only occurs at the surface of the Ni film. Actually, let it be said from the beginning, our high resolution transmission electron microscope (HRTEM) observations show that there is no graphene at the Ni$\mathrm{SiO}_{2}$ interface nor at the $\mathrm{Ni}$ grain boundaries after carbon implantation and high temperature annealing followed by

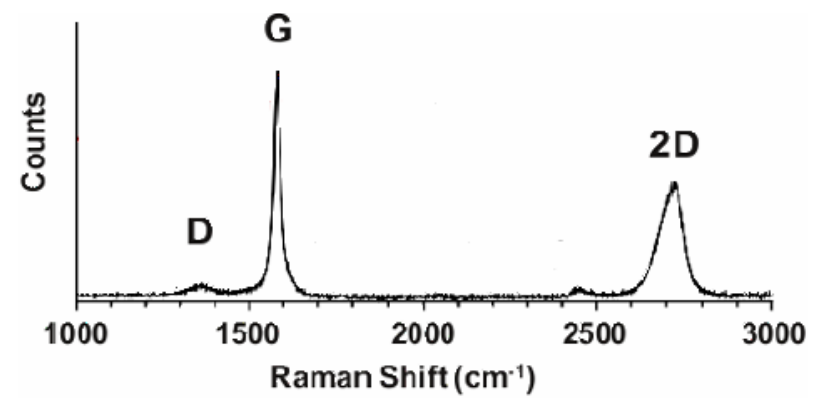

Figure 2. Raman characterization (at $514 \mathrm{~nm}$ excitation) of a graphene film still standing on top of the Ni catalyst.

Table 1. Some C solubility values in a $200 \mathrm{~nm}$ thick Ni film.

\begin{tabular}{llll}
\hline Temperature $\left({ }^{\circ} \mathrm{C}\right)$ & 725 & 900 & 1000 \\
\hline C solubility & 8 & 16 & 23 \\
$\left(\times 10^{15}\right.$ atoms cm & \\
\hline
\end{tabular}

quenching. The implanted substrates were annealed under vacuum $\left(\sim 10^{-5}\right.$ mbar $)$ using different starting and stopping temperatures (see below). The annealing was performed by pushing the samples (hosted on a quartz boat) with a stainless steel rod into the furnace which was preheated at the chosen temperature. The stainless steel rod was also used to rapidly remove the samples from the furnace, thus providing efficient quenching conditions.

From the results of Lander et al [17], we have evaluated the C solubility in a $200 \mathrm{~nm}$ thick $\mathrm{Ni}$ film at different temperatures. We have transformed the data of Lander $(\ln S=$ $2.480-4.880 / T$ where $S$ is the carbon solubility in weight $\%$ $\mathrm{C}$ into $\mathrm{Ni}$ ) into $\mathrm{C}$ atoms per $\mathrm{cm}^{3}$ of $\mathrm{Ni}$, yielding $S_{T}=$ $5.33 \times 10^{22} \exp (4880 / T)$ atoms $\mathrm{cm}^{-3}$, where $S_{T}$ is the carbon solubility expressed in atoms $\mathrm{cm}^{-3}$ at temperature $T$. From this formula, with have deduced the $\mathrm{C}$ solubility in atoms $\mathrm{cm}^{-2}$ in our $200 \mathrm{~nm}$ thick Ni films. Some values of interest for the present study are listed in table 1. In order to avoid complications with the kinetics of carbon out-diffusion from the Ni film due to the decrease of solubility as the temperature decreases [18], the following procedure was adopted after ion implantation: (i) annealing was first performed at $900^{\circ} \mathrm{C}$ over $30 \mathrm{~min}$, in order to dissolve all the implanted carbon in the Ni thin film (the carbon solubility in $\mathrm{Ni}$ at $900^{\circ} \mathrm{C}$ is $\sim 16 \times 10^{15} \mathrm{~cm}^{-2}$ - see table 1) and (ii) the furnace temperature was then slowly decreased to $725^{\circ} \mathrm{C}$ (at a rate of $0.5^{\circ} \mathrm{C} \mathrm{s}^{-1}$ ). During this temperature decrease, the carbon solubility in $\mathrm{Ni}$ evolves from $16 \times 10^{15} \mathrm{~cm}^{-2}$ down to $8 \times 10^{15} \mathrm{~cm}^{-2}$ (table 1), which means that the equivalent of $\sim 2$ graphene layers should out-diffuse and precipitate. Finally, (iii) the samples were rapidly quenched from $725^{\circ} \mathrm{C}$, thus trapping the residual carbon inside the $\mathrm{Ni}$. In this manner, we can control the amount of precipitated carbon at high temperature without knowing the details of the kinetics of carbon out-diffusion from $\mathrm{Ni}$ at moderate to low temperature.

Figure 2 shows a typical Raman spectrum taken on a sample processed as explained above (annealing at $900{ }^{\circ} \mathrm{C}$ and quenching from $725^{\circ} \mathrm{C}$ ) and still standing on top of the Ni film. It exhibits the now classical features of graphene, i.e., a small 


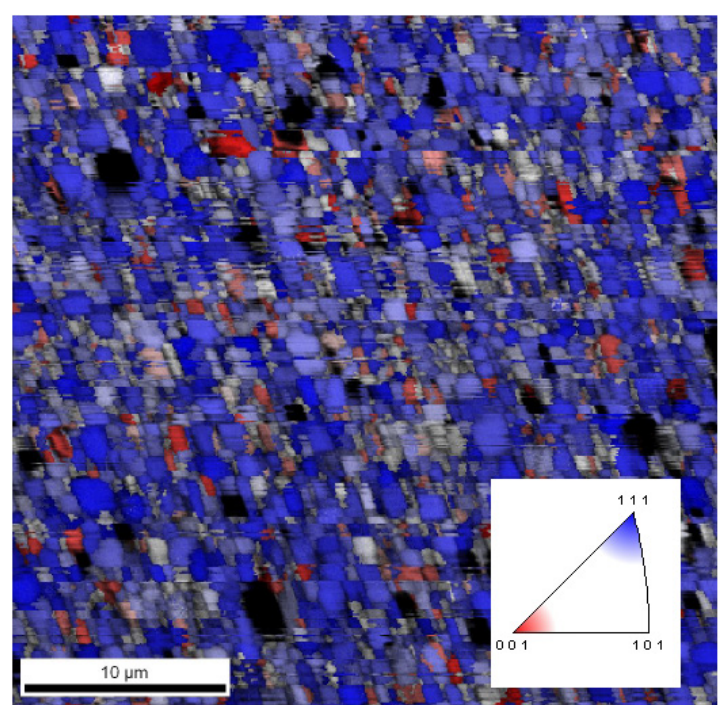

Figure 3. SEM image of a Ni film after carbon ion implantation and annealing. The local crystallographic orientation at each pixel has been measured using electron backscattering diffraction (EBSD). The dominant blue color indicates that more than $80 \%$ of the $\mathrm{Ni}$ grains are oriented within $15^{\circ}$ of $\langle 111\rangle$.

D band $\left(\sim 1350 \mathrm{~cm}^{-1}\right)$, a strong $\mathrm{G}$ band $\left(\sim 1590 \mathrm{~cm}^{-1}\right)$ and a $2 \mathrm{D}$ band $\left(\sim 2700 \mathrm{~cm}^{-1}\right)$ emerging from a double resonant scattering phenomenon [19]. The Raman shift of the 2D band $\left(2714 \mathrm{~cm}^{-1}\right)$, the high $I_{\mathrm{G}} / I_{\mathrm{D}}$ ratio (4.9) and the low $I_{\mathrm{G}} / I_{2 \mathrm{D}}$ (0.72) ratio indicate a thin layer of globally good quality graphene $[9,11,12]$. The $I_{\mathrm{G}} / I_{\mathrm{D}}$ ratio increases significantly when the graphene layers are transferred to an auxiliary substrate (see below), as also pointed out by others [20].
Next, we examined the surface structure of the $\mathrm{Ni}$ films after annealing, using electron backscattering diffraction (EBSD) in the scanning electron microscope (SEM) and cross sectional HRTEM. First of all, we observed that the Ni films were strongly (111) textured after ion implantation and annealing (figure 3). Also, the grain size was rather small, varying typically between 0.5 and $1 \mu \mathrm{m}$. Figure 4 shows a typical high resolution TEM image of the surface of a Ni grain after the annealing procedure described above. On this particular grain, which is totally covered by a rather homogeneous graphene film, we can count between 4 and 5 layers. The inset of the picture shows a higher resolution image where four graphene layers are apparent. We have additionally transferred some layers to holey carbon-coated TEM grids (figure 5). The graphene flakes appear to cover several squared micrometers, i.e., more than the typical nickel grain size. Their being folded allows one to count the number of layers on their edges: between two and four, again, in that case (figure 5). However, according to the procedure we have used for annealing, only two layers of graphene should be present on the Ni surface, assuming a uniform precipitation mechanism. Even if the solubility data of [17] are not accurate, some implanted carbon should remain trapped in the $\mathrm{Ni}$ film following the quenching step from $725^{\circ} \mathrm{C}$ and less than four graphene layers (corresponding to the total implanted dose) should appear on the Ni surface. Obviously this is not the case here, which means that the implanted carbon (whose inplane concentration was uniform after ion implantation) has been strongly redistributed upon annealing and precipitation. The strong carbon redistribution is further evidenced by the fact that in other locations (not shown here), we do not observe any graphene at all on the surface of the Ni grains, which means that the films are not continuous. It therefore seems that, during

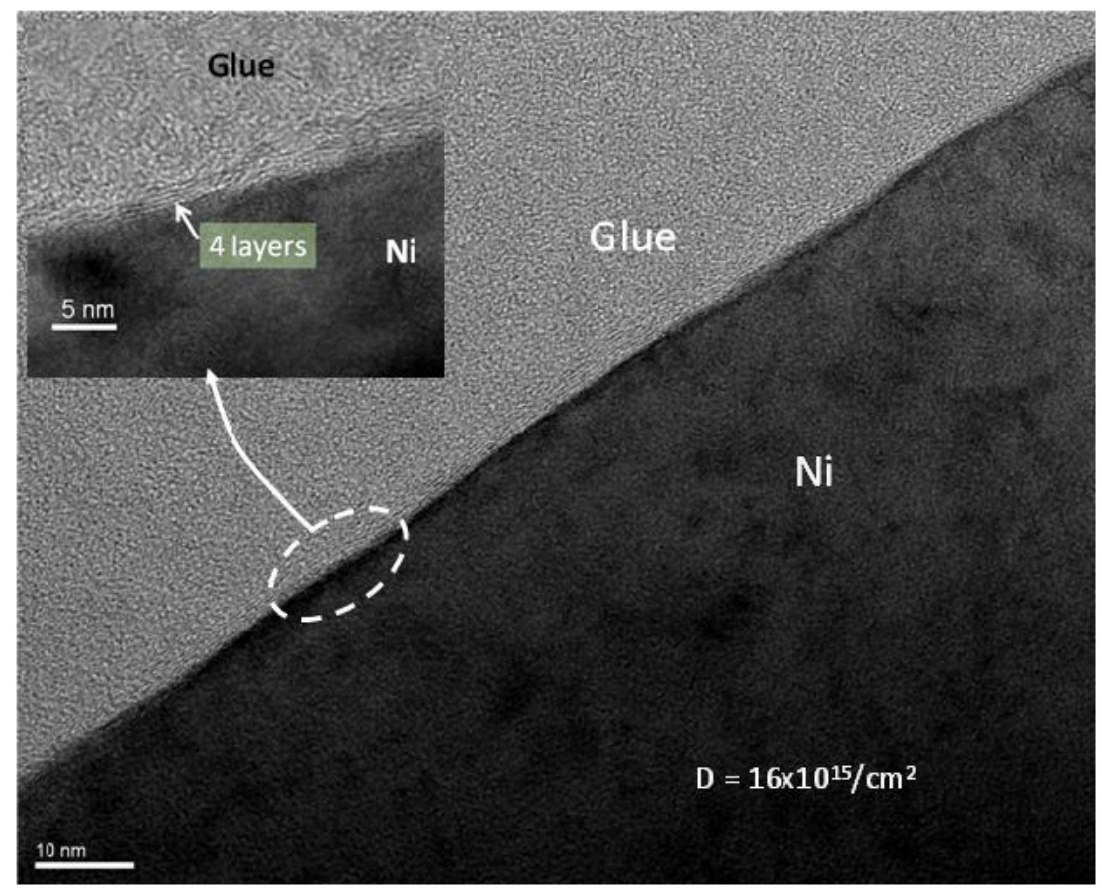

Figure 4. Cross sectional HRTEM image showing part of a graphene film with a thickness of four to five layers, extending over the whole top surface of a Ni grain. The inset is a higher magnification picture showing a particular area where four layers can be counted. 


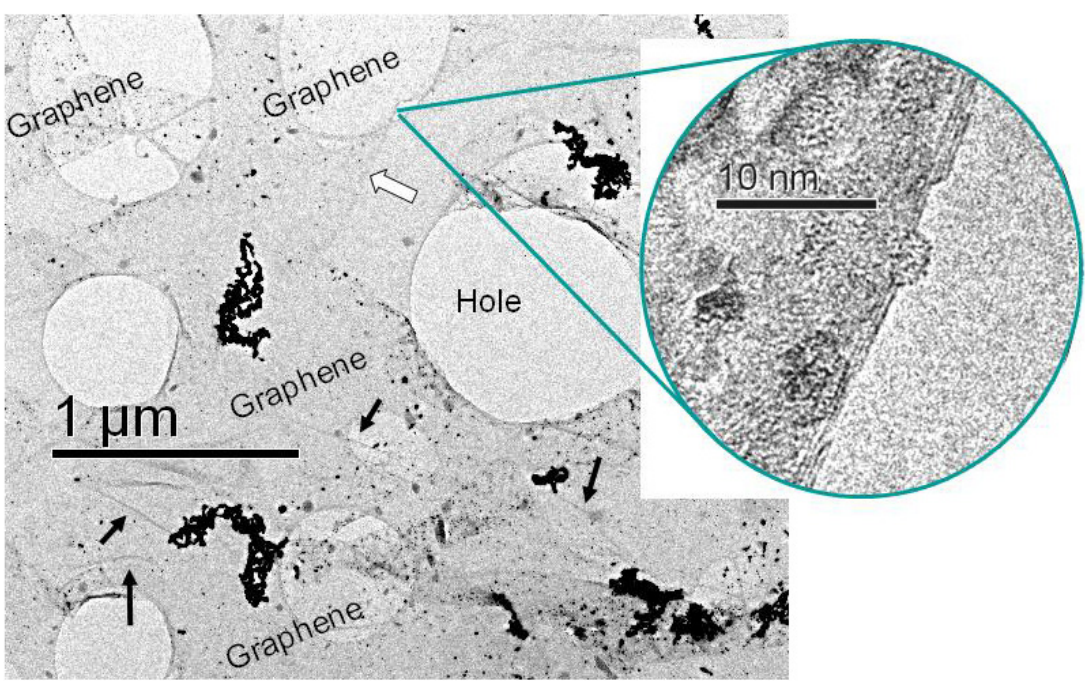

Figure 5. Plan-view TEM picture of a graphene flake, about four layers thick (inset) and exhibiting lateral dimensions larger than a micrometer. The black arrows indicate wrinkles; the white arrow shows the border of the folded flake, where the number of layers can be counted.

high temperature annealing, the implanted carbon diffuses towards dedicated nucleation sites, from where growth takes place, and that some grains are totally depleted of carbon by this diffusion process. This carbon redistribution indicates that the graphene thickness (number of monolayers) is determined by the topography of the nucleation sites (probably steps or bunches of them at the boundary of (111)-oriented Ni grains), rather than by the amount of carbon present in the Ni films, which points toward further study of the graphene nucleation mechanism on Ni thin films. This tendency of graphene to produce a non-uniform film thickness when synthesized on $\mathrm{Ni}$ has already been reported [14] but not really explained yet.

Finally, we have used the transfer length method (TLM) [21] to study the electrical properties of our few-layered graphene (FLG) films. For the transfer of FLG films onto $\mathrm{Si} / \mathrm{SiO}_{2}$ substrates, a $1.5 \mu \mathrm{m}$ thick PMMA layer was first spin coated on top of the samples after annealing. The Ni layer was then etched using a commercial etchant and the PMMA layer was dissolved once the film was deposited on the oxidized Si substrate $[8,9,12]$. The transferred FLG films were then masked and etched in an oxygen plasma and $\mathrm{Pd}$ electrodes were evaporated to a thickness of $\sim 80 \mathrm{~nm}$. A further annealing at $600^{\circ} \mathrm{C}$ under vacuum was performed in order to improve contact resistances. Figure 6 shows a scanning electron microscope (SEM) picture of a typical TLM sample on oxidized $\mathrm{Si}$. We have measured sheet resistances varying between 12 and $40 \mathrm{k} \Omega / \square$ depending on the location on the host oxidized Si substrate. These high values tend to confirm that the films are not continuous.

In conclusion, we have used the ion implantation of carbon into Ni thin films followed by high temperature annealing and quenching, in order to synthesize FLG. Our first observations indicate that the number of graphene layers in the FLG films is controlled by the graphene nucleation mechanism on Ni rather than by the amount of carbon available in the Ni film. Larger grains from Ni foils (instead of films) or pre-annealed Ni thin

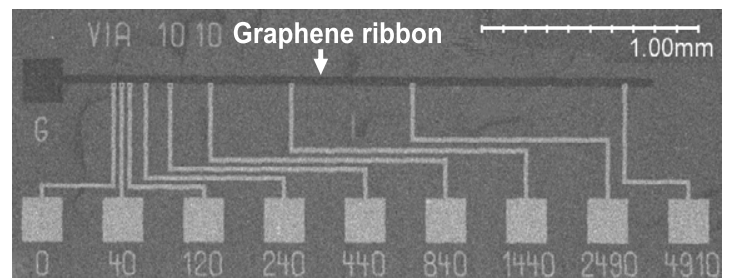

Figure 6. SEM picture of a TLM device used for the measurement of the sheet resistance of few-layered graphene films transferred on $\mathrm{Si} / \mathrm{SiO}_{2}$.

films would probably reduce the amount of nucleation centers and increase the overall uniformity of the graphene films.

\section{Acknowledgments}

DP and YHL would like to acknowledge support from the WCU program through the NRF of Korea, funded by MEST (R31-2008-000-10029-0). This work has been supported by the Region Ile-de-France in the framework of C'Nano IdF. C'Nano IdF is the nanoscience competence center of Paris Region, supported by CNRS, CEA, MESR and Region Ile-deFrance.

Note added. While this paper was in the review process, a similar one appeared [22]. The authors also point out the redistribution of carbon after annealing and cooling leading to a 'heterogeneous graphene thickness distribution on the surface of the Ni film' and they 'presume that the graphene growth is driven by the surface properties and morphology of the Ni grain', which is similar to our conclusions.

\section{References}

[1] Novoselov K S, Geim A K, Morozov S V, Jiang D, Zhang Y, Dubonos S V, Grigorieva I V and Firsov A A 2004 Electric field effect in atomically thin carbon films Science 306 666-9 
[2] Geim A K and Novoselov K S 2007 The rise of graphene Nat. Mater. 6 183-91

[3] Geim A K 2009 Graphene: status and prospects Science 324 1530-4

[4] Segal M 2009 Selling graphene by the ton Nat. Nanotechnol. 4 612-4

[5] Berger C et al 2006 Electronic confinement and coherence in patterned epitaxial graphene Science 312 1191-6

[6] Emtsev K V et al 2009 Towards wafer-size graphene layers by atmospheric pressure graphitization of silicon carbide Nat. Mater. 8 203-7

[7] Sutter P W, Flege J-I and Sutter E A 2008 Epitaxial graphene on ruthenium Nat. Mater. 7 406-11

[8] Yu Q, Lian J, Siriponglert S, Li H, Chen Y P and Pei S G 2008 Graphene segregated on Ni surfaces and transferred to insulators Appl. Phys. Lett. 93113103

[9] Reina A, Jia X, Ho J, Nezich D, Son H, Bulovic V, Dresselhaus M S and Kong J 2009 Large area, few-layer graphene films on arbitrary substrates by chemical vapor deposition Nano Lett. 9 30-5

[10] De Arco L, Zhang Y, Kumar A and Zhou C 2009 Synthesis, transfer, and devices of single- and few-layer graphene by chemical vapour deposition IEEE Trans. Nanotechnol. 8 135-8

[11] Chae S J et al 2009 Synthesis of large-area graphene layers on poly-nickel substrates by chemical vapor deposition: wrinkle formation Adv. Mater. 21 2328-33

[12] Kim K S, Zhao Y, Jang H, Lee S Y, Kim J M, Kim K S, Ahn J-H, Kim P, Choi J-Y and Hong B H 2009 Large-scale pattern growth of graphene films for stretchable transparent electrodes Nature 457 706-10
[13] Li X et al 2009 Large-area synthesis of high-quality and uniform graphene films on copper foils Science 324 1312-4

[14] Li X, Cai W, Colombo L and Ruoff R S 2009 Evolution of graphene growth on $\mathrm{Ni}$ and $\mathrm{Cu}$ by carbon isotope labeling Nano Lett. 9 4268-72

[15] Li G, Guest J R and Guisinger N P 2010 Epitaxial graphene on $\mathrm{Cu}(111)$ Nano Lett. $103512-6$

[16] The profile calculation of the implanted carbon species was performed using the SRIM program suite issued by Ziegler J F www.srim.org

[17] Lander J J, Kern H E and Beach A L 1952 Solubility and diffusion coefficient of carbon in nickel: reaction rates of nickel-carbon alloys with barium oxide J. Appl. Phys. 23 1305-9

[18] Wiltner A, Linsmeier Ch and Jacob T 2008 Carbon reaction and diffusion on $\mathrm{Ni}(111), \mathrm{Ni}(100)$, and $\mathrm{Fe}(110)$ : kinetic parameters from $\mathrm{x}$-ray photoelectron spectroscopy and density functional theory analysis $J$. Chem. Phys. 129084704

[19] Ferrari A C et al 2006 Raman spectrum of graphene and graphene layers Phys. Rev. Lett. 97187401

[20] Li X, Zhu Y, Cai W, Borysiak M, Han B, Chen D, Piner R D, Colombo L and Ruoff R S 2009 Transfer of large-area graphene films for high-performance transparent conductive electrodes Nano Lett. 9 4359-63

[21] Berger H H 1972 Models for contacts to planar devices Solid-State Electron. 15 145-58

[22] Garaj S, Hubbard W and Golovchenko J A 2010 Graphene synthesis by ion implantation Appl. Phys. Lett. 97183103 